\title{
Friction of Self-Lubricating W-S-C Sputtered Coatings Sliding Under Increasing Load
}

\author{
Tomas Polcar, Manuel Evaristo, Albano Cavaleiro*
}

W-S-C films were deposited by non-reactive magnetron sputtering from a carbon target with several pellets of $\mathrm{WS}_{2}$ incrusted in the zone of the preferential erosion. The number of the pellets was changed to modify the carbon content in the films, which varied from 26 up to 70 at.-\%. Alloying W-S films with carbon led to a substantial increase in the hardness in the range of 4-10 GPa; the maximum hardness was obtained for the coatings with carbon contents close to 40 at.-\%. XRD diffraction patterns showed that there was a loss of crystallinity with the increase of the carbon content in the film. C-contents in the range (37-66 at.-\%) were selected for sliding tests (pin-on-disk, $100 \mathrm{Cr} 6$ steel ball as a counterpart) carried out in humid air under increasing contact load. The friction coefficient was observed to decrease continuously with increasing load. The wear tracks and wear debris were also analyzed by Raman spectroscopy to understand the structural transformations induced by the increasing load. The friction results were compared with existing models for pure transition metal dichalcogenides (TMD), and it could be concluded that the friction mechanisms of W-S-C coatings fundamentally differ from those of pure TMD.

\section{Introduction}

Transition metal dichalcogenides (TMD) have excellent self-lubricant properties in dry air or vacuum; however, these films are easily rusted in the presence of moist environments and their reduced mechanical resistance makes them inappropriate for applications requiring high load bearing capacity. On the other hand, hard coatings are often employed to protect parts from abrasive and erosive wear; however, they do not have low friction and they are frequently brittle. Therefore, there is a need to design coatings combining low friction with high load-bearing capacity; moreover, a good adhesion to the substrates is required. Our recent works showed that the synergetic effect of doping W-S films with carbon or nitrogen together with the deposition of a Ti interlayer could give rise to significant improvements in the mechanical properties

T. Polcar, M. Evaristo, A. Cavaleiro

ICEMS - Faculdade de Ciências e Tecnologia da, Departamento de Engenharia Mecânica, Universidade de Coimbra, Rua Luís Reis Santos, Coimbra 3030-788, Portugal

Fax: (+351) 239790 701; E-mail: albano.cavaleiro@dem.uc.pt and the tribological behavior, particularly in the case of films alloyed with carbon. ${ }^{[1,2]}$

The objective of this work was to develop coatings capable of being used in a wide rage of applications, with different applied loads, temperatures and environments. In this study, the structural and mechanical properties of W-S-C coatings are presented together with their sliding properties under different load conditions.

\section{Experimental Part}

\begin{abstract}
All W-S-C coatings were deposited on $100 \mathrm{Cr} 6$ and M2 polished steel samples with hardness close to 5 and $9 \mathrm{GPa}$, respectively. The depositions were carried out in a radio-frequency magnetron sputtering Edwards ESM 100 unit, equipped with two cathodes $(\varnothing=100 \mathrm{~mm})$. Prior to the depositions, the substrates were sputter cleaned for $20 \mathrm{~min}$ by establishing the plasma close to the substrates electrode. Immediately after, a $\mathrm{Ti}$ interlayer was deposited with an approximate thickness of $300 \mathrm{~nm}$. The main sputtering target was pure carbon, partly covered by $\mathrm{WS}_{2}$ pellets placed preferentially in the eroded zone. The dimensions of the pellets were $4.1 \times 3.5 \times 1.5 \mathrm{~mm}$. The degree of target coverage
\end{abstract}


determined the overall chemical composition of the sputtered film. The depositions were carried out with constant power density of $7.6 \mathrm{~W} \cdot \mathrm{cm}^{-2}$ in the carbon target.

To evaluate the chemical composition of the films, a Cameca SX 50 electron probe microanalysis was used. The hardness was determined by a depth-sensing indentation technique using a Fisherscope H100; the coatings deposited on M2 steel were used for these measurements.

Tribological tests were carried out with a pin-on-disk tribometer (CSM Instrument). $100 \mathrm{Cr} 6$ steel balls were used as sliding partners. The diameter of the ball was $6 \mathrm{~mm}$, load was in the range of 5-47 N. The tribological behavior of the coatings was evaluated in air with a relative humidity of $30 \%$, the sliding speed was 0.05 $\mathrm{m} \cdot \mathrm{s}^{-1}$. The friction coefficient reported in this study is the average value of the whole sliding test, unless stated otherwise.

\section{Results and Discussion}

\section{Chemical Composition, Structure, and Mechanical Properties}

Different carbon contents were achieved in the coatings by varying the number of $\mathrm{WS}_{2}$ pellets in the $\mathrm{C}$ target. As it was expected, the carbon content of the films decreased almost linearly with the increase in the total area of pellets. The chemical composition of the deposited coatings varied from 29 to 70 at.-\% C (hereinafter, the denomination "at.-\% C" represents the carbon content in the coating). The S/W ratio varied in the range of $1.2-1.5$, a slight increase was observed with increasing carbon content. The sulfur deficiency is probably caused by either the resputtering of sulfur atoms from the growing film by energetic neutrals or by the chemical reactions of sulfur with the residual atmosphere.

X-ray diffraction patterns of W-S-C films showed a gradual loss of crystallinity with the increase of the carbon in the coatings. The films with a low carbon content present typical XRD patterns of Me-S (Me, transition metal) sputtered films with prominent peaks at $2 \theta \approx 40^{\circ}$ with an extended shoulder corresponding to a turbostrating stacking of (10L) planes $(L=0,1,2,3)$, and $2 \theta \approx 70^{\circ}$ indexed as (110) planes. Wiese et al. ${ }^{[3]}$ demonstrated that these XRD patterns could be explained by a $2 \mathrm{D}$ organization of the basal planes which could have a dimension of several tens of unit cells. With the progressive decrease of the lateral dimensions of the basal planes, either broadening or drop in the intensity of the (10L) plan occurred until a unique low intensity and broad peak typical of an amorphous structure was detected. This situation would arise when the lateral order of the basal planes did not exceed a couple of lattice parameters. ${ }^{[3]}$ In previous work, ${ }^{[4]}$ it was shown by HRTEM that W-S-C films with high $\mathrm{C}$ contents were formed by a nanocomposite structure which included $\mathrm{WS}_{2}$ grains of a few nanometers size, in agreement with Wiese et al.'s interpretation.
The hardness of W-S-C films increased with increasing carbon content to reach a maximum at $\sim 40$ at.- $\%$ C (10 GPa). Further increase of the carbon content led to smaller values of the hardness. This trend can be related to the synergetic actions of the compactness increase of the films associated with the possible formation of nano-sized carbide phases, intrinsically harder than tungsten disulfide. With the addition of more carbon to the films, there is no more $W$ available to establish $\mathrm{W}-\mathrm{C}$ bonds and the formation of carbon phases leads to a decrease of the hardness (see Figure 1). It should be remarked that with the deposition conditions used in this work, a pure C film exhibited only about $8 \mathrm{GPa}$ hardness. However, the hardness of W-S-C coatings is generally about one order of magnitude higher than that of pure tungsten disulfide. ${ }^{[5]}$

\section{Friction and Wear Properties}

The first set of sliding tests was carried out with 500 laps. The depth of the wear track was very low to calculate the wear rate and, thus, selected coatings were tested with 5000 laps. The average friction coefficient of the coatings as a function of the load is shown in Figure 2. As a general trend, the friction coefficient decreases with increasing load. Nevertheless, there is significant difference between the values corresponding to 500 and 5000 laps. A detailed analysis of the friction curves revealed two different typical trends: (i) the coatings up to 51 at.-\% C exhibited high friction in the first hundred laps (running-in), which was followed by a drop in the friction to a lower level corresponding to the steady state wear; (ii) the coatings with the highest carbon contents showed a higher friction coefficient, which remained almost constant during the entire test. As a consequence, the average friction for the

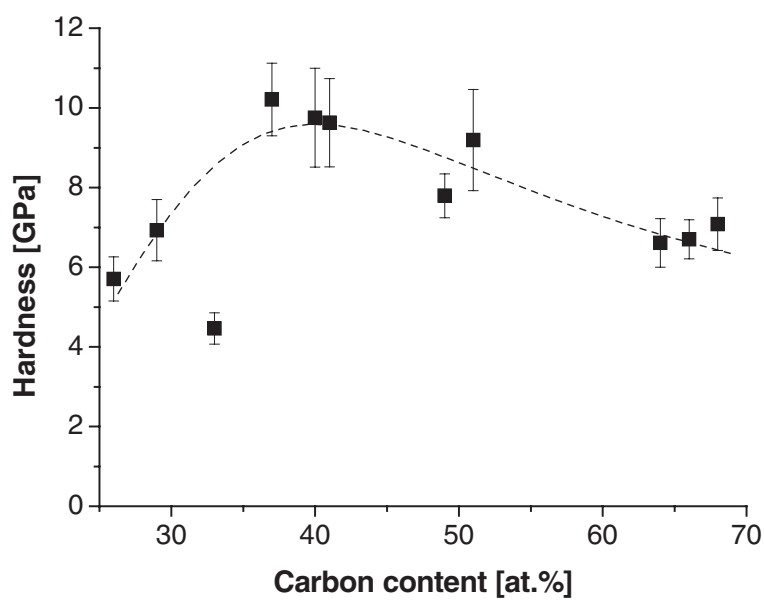

Figure 1. Evolution of the hardness with the carbon content in the films. The dashed line is a guide to the eye. 


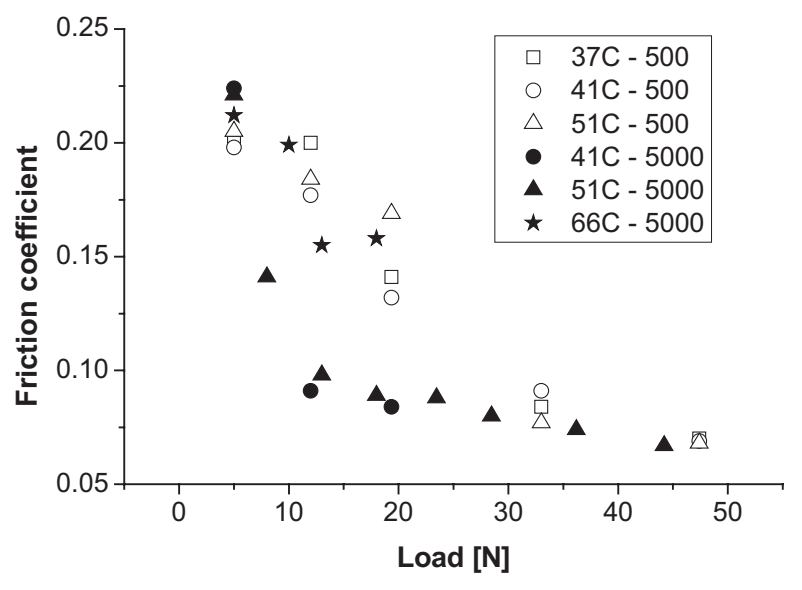

Figure 2. Friction coefficient of W-S-C coatings as a function of the load (note the different number of laps).

low and medium carbon content coatings decreases with the number of laps, while it remains the same in the case of high carbon content.

The wear tracks produced by the sliding were very shallow for 500 laps tests (typically less than $200 \mathrm{~nm}$ ), which gives rise to very small worn volumes and, consequently, wear rate calculations are very difficult. Therefore, we can report only on the wear rate of the coatings which underwent tests with 5000 laps. The wear rate of the 51 at. $\%$ C coating initially increased from $0.55(5 \mathrm{~N})$ to $1.36 \times 10^{-6}(18 \mathrm{~N})$ and than slowly decreased to $0.93 \times$ $10^{-6}$, while the 41 at.-\% C coating (tested up to $19 \mathrm{~N}$ load) showed rather constant low wear rates, i.e., a small increase from $0.60(5 \mathrm{~N})$ to $1.06 \times 10^{-6}(19 \mathrm{~N})$

\section{Analysis of the Worn Surfaces}

The balls after the tests were covered by an adhered layer of the material transferred from the coating. When the adhered layer was removed, the optical observation revealed no wear scar on the ball. Chemical maps of this layer showed an almost homogeneous distribution of W, S, $\mathrm{C}$, and $\mathrm{O}$. No vestiges of $\mathrm{Fe}$ originating from steel were observed, which confirms that the ball wear rate was negligible.

The wear tracks of the coatings with carbon content up to 51 at.-\% observed by SEM were very smooth with no significant scratches even when the highest load was applied. The EDX analysis of the center of the wear tracks showed the same chemical composition as the unworn surface. However, at higher loads exceeding $20 \mathrm{~N}$, the sides of the wear tracks were covered by adhered wear debris rich in tungsten and oxygen. The wear tracks produced by sliding on the high carbon content coatings were covered

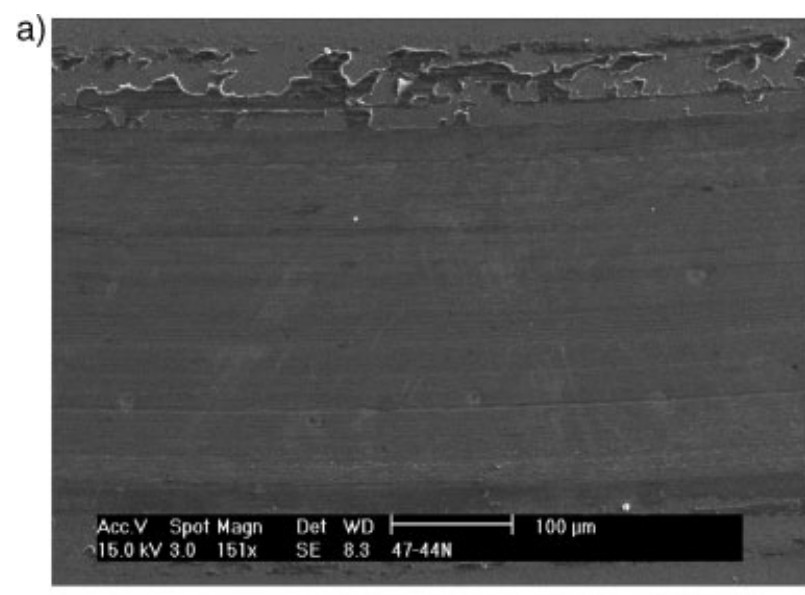

b)

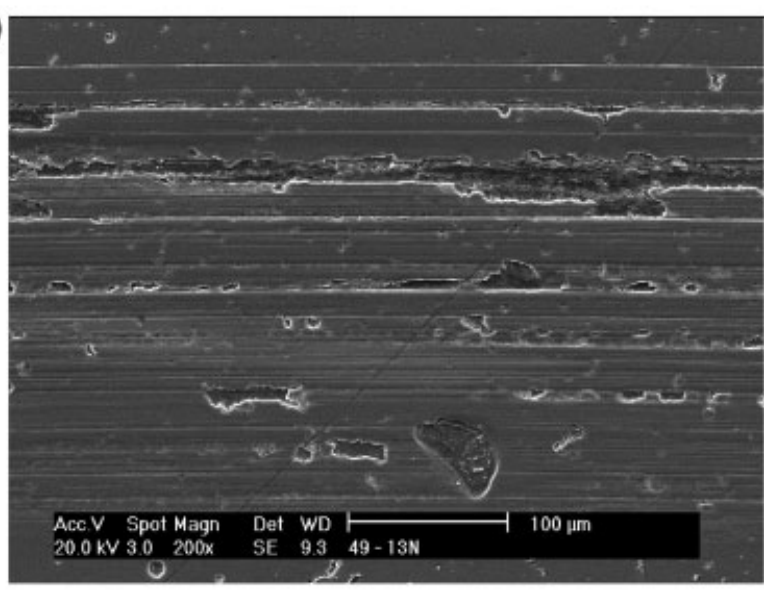

Figure 3. SEM micrographs of the wear tracks: (a) coating with 51 at.-\% C, load $44 \mathrm{~N}$ and (b) 66 at.-\% C, $13 \mathrm{~N}$.

by scratches parallel to the relative movement of the ball. The wear debris layer formed isles adhered on the worn surface of the coating (Figure 3).

Raman spectroscopy was carried out in different places in the wear tracks and outside. Two groups of peaks could be observed, one corresponding to carbon (i.e. D and G peaks) and another consisting of two peaks (325 and $\left.416 \mathrm{~cm}^{-1}\right)$. The later group was identified as $\mathrm{WS}_{2}$, since both peaks match well to the Raman spectrum taken from a $\mathrm{WS}_{2}$ pellet. Unfortunately, the laser power had to be lowered to very low values in order to avoid the coating damage, predominantly graphitization, which could be observed for higher power values. Consequently, the obtained spectra were very noisy and their deconvolution difficult. However, an interesting feature was observed, the ratio $I_{\mathrm{c}} / I_{\mathrm{Ws} 2}$, calculated as the sum of the carbon peaks areas divided by the sum of the $\mathrm{WS}_{2}$ peaks areas, decreased from the unworn surface to the center of the wear track. This behavior is typical for all coating compositions; an example is shown in Figure 4. 


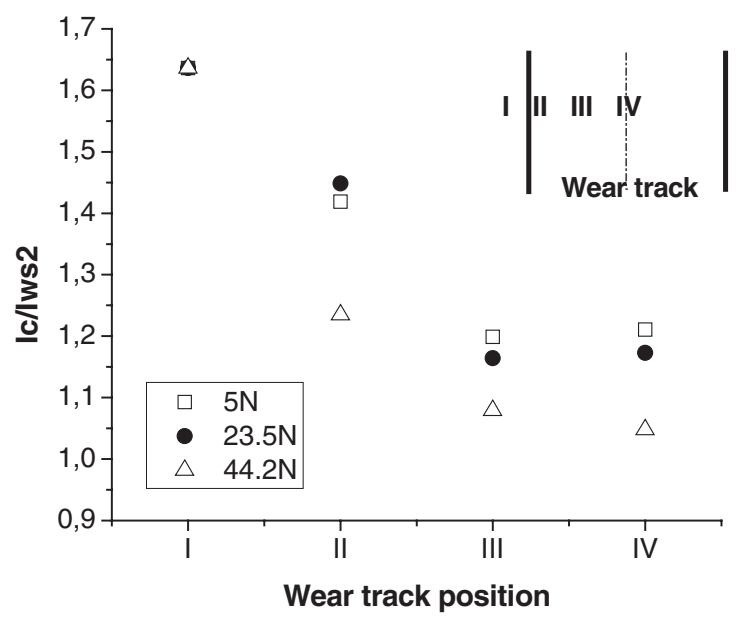

Figure $4 . I_{\mathrm{c}} / I_{\mathrm{WS} 2}$ ratio for different loads as a function of the position in the wear track, coating with 51 at.-\% C.

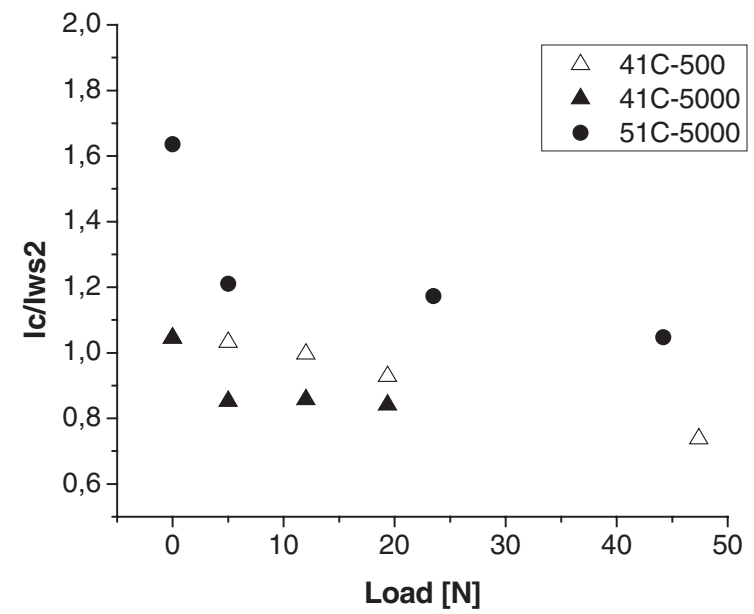

Figure $5 . I_{C} / I_{\text {WS2 }}$ ratio as a function of load for coatings with 41 and 51 at.-\% C. Note the different number of laps, 500 and 5000.

\section{Frictional Mechanism}

The results of the frictional analysis complemented by the observation of the worn surfaces show that the third body formed between the two surfaces in the contact plays a dominant role in the friction process. Only coatings with a carbon content up to 51 at. $\%$ C were selected for further analysis of the frictional mechanisms, since the third body was not homogeneous in the case of the wear tracks produced on the coating with the highest carbon content. $\mathrm{Wu}$ et al. tested W-S-C coatings with similar chemical composition sliding in air and they concluded that the development of a uniform well-bonded layer on the pin was associated to the low friction. ${ }^{[6]}$ They observed no surface layer on the coating after the test and their Raman spectrum obtained in the wear track was identical with that of the unworn surface. However, as referred to above, significant changes in the Raman spectra taken from different parts of the wear tracks were observed in this work.

The decrease in the $I_{\mathrm{c}} / I_{\mathrm{WS} 2}$ ratio towards the center of the wear track presented in Figure 4 indicates that an increasing contact pressure induces the formation of a thin tungsten sulfide film on the coating surface, or a structural change in the bulk of the coating, or a combination of both. The $I_{\mathrm{c}} / I_{\mathrm{WS} 2}$ ratio obtained from the Raman spectra taken from the center of the wear tracks of a selected coating is lower in the case of tests with higher number of laps, see Figure 5. Therefore, whatever the change in the coating induced by sliding is, it is enhanced by the increasing number of laps.

The decrease of the friction with increasing load of W-S-C coatings is very similar to the frictional behavior of pure TMD coatings. ${ }^{[7-9]}$ The easy intra- and intercrystalline slip in the friction contact due to weak Van der Waal's forces between the lamellae of S-W-S hexagonal basal planes of pure tungsten disulfide facilitates the friction by diminishing the tangential force necessary for the sliding. During a pin-on-disk test, the transfer of the coating material to the ball surface and the reorientation induced by the friction of the randomly oriented $\mathrm{WS}_{2}$ grains, in the contact area, to the basal plane orientation, transforms the materials in contact. Consequently, the friction force that originated in this case is due, predominantly, to the slipping referred to above. Zabinski et al. ${ }^{[10]}$ showed, for the W-S-C system with 20 at.-\% of sulfur content that the friction mechanism intervening in the sliding contact was very similar to that of pure tungsten disulfide and that the contribution of the carbon to the friction was negligible.

Therefore, the methodology used for the analysis of the frictional properties of pure TMD coatings, particularly for $\mathrm{MoS}_{2}$ coatings, has been used for W-S-C coatings as a function of the load.

\section{Frictional Heating}

The friction coefficient is significantly higher when TMD materials are sliding in humid air than in dry air. The heating of the TMD surface during sliding causes the drying of the air and, consequently, the decrease in the friction. ${ }^{[11]}$ Therefore, if the increase in the contact pressure causes an increase in the temperature of the coating surface, the friction should diminish. The rate of the frictional heating $\left(R_{\mathrm{FH}}\right)$ per unit sliding area can be derived as

$$
R_{\mathrm{FH}}=\frac{P_{\mathrm{FC}}}{2 a l}
$$


where $a$ is the Hertzian radius ${ }^{[12]}, l$ the sliding distance, and $P_{\mathrm{FC}}$ the rate of the frictional heating calculated as

$$
P_{\mathrm{FC}}=\mu L S
$$

where $\mu$ is the friction coefficient, $L$ the normal load, and $s$ the sliding speed. The calculations show that there is no significant increase in $R_{\mathrm{FH}}$ with increasing normal load. Therefore, the drying of the air due to the frictional heating does not contribute to the observed decrease in the friction with load.

\section{Shear Stress Contribution}

For $\mathrm{MoS}_{2}$ coatings, the Hertzian contact model was used to explain the frictional behavior for a pin-on-disk configuration. The model was derived from the formula approximating the shear stress of solids at high pressures as

$$
\tau=\tau_{0}+\alpha P
$$

where $\tau$ is the shear strength and $P$ is the contact pressure. ${ }^{[13]}$ The constants $\tau_{0}$ and $\alpha$ are material properties. Dividing Equation 3 by the contact pressure calculated as the mean Hertzian pressure ${ }^{[14]}$ gives

$$
\mu=\tau_{0} \pi\left(\frac{3 R}{4 E}\right)^{\frac{2}{3}} L^{-\frac{1}{3}}+\alpha
$$

where $R$ is the radius of the ball, $L$ the normal load, and $E$ the composite modulus of the couple given by

$$
\frac{1}{E}=\frac{1-v_{1}^{2}}{E_{1}}+\frac{1-v_{2}^{2}}{E_{2}}
$$

where $E_{1,2}$ and $v_{1,2}$ are the Young's moduli and the Poisson ratios of the coating and the ball. The experiments showed that the described model can be used as a good approximation to explain the decrease in the friction of $\mathrm{MoS}_{2}$ with the contact pressure. Singer et al. used the power analysis applied to Equation 4 satisfying $L^{-1 / 3}$ behavior, which allowed to calculate $\alpha$ as $0.001 \pm 0.001 .^{[8]}$ Grosseau-Poussard et al. used a linear analysis considering $\alpha$ being close to zero and they too confirmed $L^{-1 / 3}$ behavior. ${ }^{[14]}$

However, the calculations of the present research show that the referred approach is not applicable for W-S-C coatings. The power analyses applied to Equation 4, where power over $L$ and $\alpha$ were variables, give as the best fit values -0.54 and a negative $\alpha$. Therefore, Equation 4 is not applicable to this case. This result was also confirmed by the examination of the $\log (\mu)$ against $\log (L)$ plots.

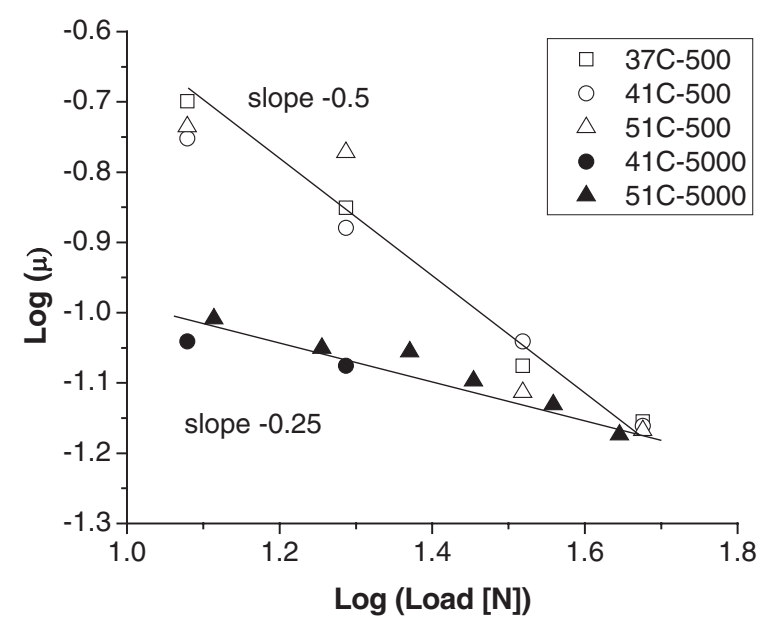

Figure 6. Log(friction) versus $\log (\mathrm{load})$. Straight lines are the least square fits of all points corresponding to 500 and 5000 laps, respectively.

\section{Mechanisms of Friction of W-S-C Coatings}

The formation of a thin surface layer on both surfaces in the contact significantly decreases the friction coefficient. This formation corresponding to the running-in process is connected with a high friction coefficient and the polishing of asperities in the contact. However, a minimal $\mathrm{W}$-S content in W-S-C coatings is required to decrease the friction. The coatings with high carbon content, i.e. with 66 at.-\%, exhibit no running-in period and their friction remained high during the entire test. The evolution of the friction coefficient with the load of W-S-C coatings up to 51 at. $\% C$ is very similar to that of pure TMD and no significant differences among various chemical compositions have been found. Since Raman spectroscopy has shown an increase of W-S to carbon ratio in the wear track, the formation of a thin $\mathrm{WS}_{2}$ layer, supposedly with (002) orientation, should be expected. However, the shear stress analysis has shown that the frictional process of W-S-C coatings is not fully comparable to that of TMD. Therefore, the intra- and intercrystalline slip between S-W-S lamellae is not the exclusive frictional mechanism.

Nevertheless, the evolution of the friction coefficient with load shows other interesting features, since a linear dependence of $\log (\mu)$ with $\log (L)$ has been obtained (Figure 6). It seems that the friction coefficient can be calculated as a function of the load by a simple empirical equation

$$
\mu=A L^{B}
$$

where $A$ is a constant corresponding to the material and the contact conditions, and the constant $B$ represents the influence of the number of laps, i.e. the formation of a third 
body and/or the structural changes of the coating in the contact area. This behavior is independent of the carbon content of the coating in the range 37-51 at.-\%.

\section{Conclusion}

W-S-C coatings deposited by rf magnetron sputtering from a carbon target embedded with a different number of $\mathrm{WS}_{2}$ pellets were tribologically tested under different loads in humid air. The average friction coefficient generally decreased with the increase of the load reaching values as low as 0.05 . Raman spectroscopy analysis revealed a structural change of the coating in the contact area. The decrease of the friction with increasing load is not connected with the frictional heating, since the rate of the frictional heating per unit sliding area remained almost constant regardless of the contact pressure. Despite the similarity in the frictional behavior of W-S-C coatings and pure TMD, the friction mechanism is different.

Acknowledgements: The authors would like to acknowledge the financial support by FCT (Project POCTIEME 46548/2002).

Received: September 11, 2006; Revised: November 21, 2006; Accepted: December 4, 2006; DOI: 10.1002/ppap.200731402
Keywords: hardness; magnetron sputtering; self-lubricant; thin films; tribology; WSC

[1] A. Nossa, A. Cavaleiro, Surf. Coat. Technol. 2001, 142-144, 984.

[2] A. Nossa, A. Cavaleiro, Surf. Coat. Technol. 2003, 163-164, 552.

[3] G. Wiese, N. Mattern, H. Herman, A. Teresiak, I. Bascher, W. Bruckner, H.-D. Bauer, H. Vinzelberg, G. Reiss, U. Kreissig, M. Mader, P. Markschlager, Thin Solid Films 1997, 298, 98.

[4] A. Nossa, A. Cavaleiro, N. J. M. Carvalho, B. J. Kooi, J. Th. M. De Hosson, Thin Solid Films 2005, 484, 389.

[5] S. Watanabe, J. Noshiro, S. Miyake, Surf. Coat. Technol. 2004, 183, 347.

[6] J.-H. Wu, M. Sanghavi, J. H. Sanders, A. A. Voevodin, J. S. Zabinski, D. A. Rigney, Wear 2003, 255, 859.

[7] B. J. Briscoe, A. C. Smith, ASLE Trans., 25, 349.

[8] I. L. Singer, R. N. Bolster, J. Wegand, S. Fayeulle, B. C. Stupp, Appl. Phys. Lett. 1990, 57, 995.

[9] A. K. Kohli, B. Prakash, Tribol. Trans. 2001, 44, 147.

[10] A. A. Voevodin, J. P. O'Neill, J. S. Zabinski, Surf. Coat. Technol. 1999, 116-119, 36.

[11] T. Kubart, T. Polcar, L. Kopecky, R. Novak, D. Novakova, Surf. Coat. Technol. 2005, 193, 230.

[12] W. C. Young, "Roark's Formulas for Stress and Strain", MCGraw Hill, New York 1989.

[13] L. D. Towle, J. Appl. Phys. 1971, 42, 2368.

[14] J. L. Grosseau-Poussard, P. Moine, M. Brendle, Thin Solid Films 1997, 307, 163. 